\section{Noninvasive Measurements of Pressure for Detecting Compartment Syndromes}

\begin{abstract}
Background: We tested a noninvasive ultrasound, Pulse Phase Locked Loop (PPLL) technique for estimating Intramuscular Pressure (IMP) in a model Acute Compartment Syndrome (ACS); and compared it to a Near-Infrared Spectroscopy (NIRS) method.

Questions: We wanted to validate our model compartment syndrome, and to compare and validate the PPLL and NIRS methods of detecting compartment syndrome.

Methods: To simulate the tamponade of an ACS, external-pressure levels from 10 to $70 \mathrm{~mm} \mathrm{Hg}$ were applied to the legs of 15 healthy adult subjects to raise their IMP. Receiver Operator Characteristic (ROC) curves were used to determine the sensitivity and specificity of diagnosing elevated IMP by the two noninvasive techniques.

Results: NIRS data varied significantly with compression $(p=0.003)$ with large subject-to-subject variability $(p<0.001)$. PPLL data also varied significantly with compression $(p=0.004)$, but subject-to-subject variation was not significant $(p=0.47)$, suggesting that individual variation does not affect the diagnostic accuracy of the PPLL technique. Sensitivity and specificity for diagnosing normal IMP by the PPLL ( $<30 \mathrm{~mm} \mathrm{Hg}$ from a slit catheter reading) from elevated IMP (>30 mm Hg) were 0.75 and 0.75 , respectively, and the area under the curve (AUC) was 0.78 . For the NIRS, the sensitivity and specificity were 0.65 and 0.65 , respectively, and the AUC was 0.68
\end{abstract}

Conclusions: Both NIRS and PPLL recordings are able to differentiate a simulated ACS up to $70 \mathrm{~mm} \mathrm{Hg}$. However, the PPLL technique is a slightly better diagnostic predictor than NIRS with less subject-tosubject variability and slightly better sensitivity and specificity.

Level of Evidence: Level II, Diagnostic test.

\section{Introduction}

Acute compartment syndrome (ACS) is a condition which occurs when increased intramuscular pressure (IMP) within a closed fascial space impairs vascular perfusion to a point below the level necessary for muscle and nerve viability. ACS can be diagnosed based on clinical findings such as the 6 Ps: pressure, pain, paresthesias, paresis, pink skin, and presence of a distal pulse [1]. However, these clinical findings have a low positive predictive value and a high negative predictive value, so the absence of the clinical findings is more useful in excluding the diagnosis of ACS than in making a diagnosis [2] A direct IMP measurement is an important adjunct in making a diagnosis for undependable and ambiguous clinical presentations, such as in cases of severe trauma, unconscious patients, or children.

Various criteria are used for diagnosis of ACS, including an absolute IMP level higher than normal $(>30 \mathrm{~mm} \mathrm{Hg}$ ) with clinical symptoms [1], or a tissue perfusion pressure $(\Delta \mathrm{P}=$ mean arterial pressure MAP - IMP) of less than 30-40 $\mathrm{mm} \mathrm{Hg}$ [2]. Currently, a direct IMP measurement is accepted as the gold standard for diagnosis by most clinicians, but requires invasive monitoring of the compartment with a slit or transducer-tipped catheter [3]. While the technique is generally accurate and reproducible, several factors such as pain,

\section{Journal of}

Orthopedics \& Rheumatology

\section{Samantha H. Lee ${ }^{1 *}$, Michael Padilla², John E. Lynch $^{3}$ and Alan R. Hargens ${ }^{2}$}

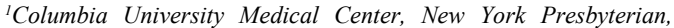
Department of Anesthesiology, New York, 10032, USA

${ }^{2}$ University of California, San Diego, Department of Orthopedic Surgery, San Diego, California 92103, USA

${ }^{3}$ CIRS, Inc., 2428 Alameda Ave Ste 316, Norfolk, Virginia 23513, USA

\section{*Address for Correspondence}

Samantha H Lee, Department of Anesthesiology, Columbia University Medical Center, New York Presbyterian, New York, 10032, USA, E-mail: shl9069@nyp.org

Submission: 24 October 2013

Accepted: 18 December 2013

Published: 21 December 2013

Copyright: ๑ 2013 Lee SH, et al. This is an open access article distributed under the Creative Commons Attribution License, which permits unrestricted use, distribution, and reproduction in any medium, provided the original work is properly cited.

Reviewed \& Approved by: Dr. Martin Grabois

Department of Physical Medicine and Rehabilitation, Baylor College of Medicine, USA

infection risk, and disagreement over the appropriate threshold pressure for diagnosis of ACS leaves some clinicians reluctant to perform direct IMP measurements. Thus, the development of a noninvasive, reproducible, and accurate method to monitor IMP in suspected ACS would ameliorate some of these uncertainties, as well as provide a new, practical tool for use in field situations where access to invasive measurement is limited.

Treatment of ACS relies on early recognition and timely fasciotomy to prevent irreversible muscle death and other complications, such as contracture or a devascularized limb requiring amputation. Thus, development of a reliable noninvasive technique could address misgivings about invasive measurement and reduce ambiguities of clinical diagnosis which could otherwise delay treatment.

A recently developed noninvasive alternative was first described by Lynch et al. [3], in which the ultrasonic pulsed phased locked loop (PPLL) was used to measure micron-level tissue displacements, which later translated to the detection of arterial pulsations within a muscle compartment. The automated analysis PPLL estimates IMP without calibration [2]. A more recently-developed digital implementation of the PPLL measures the ultrasonic phase shift more accurately, and may differentiate between displacements caused by the surrounding skin and fat between the transducer and fascia or the displacements of specific regions of the compartment.

Non-invasive NIRS is also proposed as a method of detecting ACS [4]. It is suggested that tissue oxygenation decreases significantly with decreasing limb perfusion, thus differentiating between patients with ACS and those without ACS. However, there are variables that may affect the efficacy and accuracy of these measurements, such as low oxygen tissue saturation globally, or variability in the sizes and anatomy of subjects' limbs [5]. 
Citation: Lee SH, Padilla M, Lynch JE, Hargens AR. Noninvasive Measurements of Pressure for Detecting Compartment Syndromes. J Orthopedics Rheumatol. 2013;1(1): 5.

In this study, we have developed a novel way to simulate elevated IMP based on previous models including Styf and co-worker's leg elevation [6] and Reneman's leg chamber pressure [7]. Also, we use a newly developed digital implementation of the PPLL (dPPLL) ENTACT $^{\mathrm{TM}}$ provided by Luna Innovations [8]. The dPPLL converts the phase measurements into compartment distance displacements by assuming that ultrasonic velocity is constant [9]. The goal of this study is to compare the dPPLL and NIRS measurements with direct IMP using an invasive slit catheter technique. In this regard, we hypothesize that the dPPLL can be used to detect elevated IMP in this model with higher sensitivity and specificity than that of the NIRS.

\section{Materials and Methods}

\section{Subjects}

Subjects were recruited by posting flyers in the community. Subjects had to be in good health, could withstand the experiment, and did not have any sign of infections over the catheter insertion site. Subjects were not selected based on body habitus, and subjects' BMI ranged from 20-35. Subjects were provided with information about the study, goals of the study, what would be done, and questions were answered accordingly. The subjects provided signed consent forms as approved by the institutional review board (IRB 081820).

\section{Compartment syndrome model}

In this compartment syndrome model, the subject placed his or her leg up to the thigh area into a 12" x 36" x 12" $(30.5 \mathrm{~cm}$ x $91.4 \mathrm{~cm}$ $\mathrm{x} 30.5 \mathrm{~cm}$ ) chamber. The chamber had an opening which was sealed by a neoprene sleeve, made to fit snugly around the subjects' thighs. A vacuum cleaner's exhaust was attached to the chamber so as to pump air in, controlled by a variable power rheostat, and pressures in the chamber from zero to $70 \mathrm{~mm} \mathrm{Hg}$ were measured by the pressure monitor. $70 \mathrm{~mm} \mathrm{Hg}$ was selected as the upper limit as this was a pressure that could be safely created by equipment without excessive discomfort to the subject.

\section{PPLL model}

In this digital implementation of the EN-TACT ${ }^{\mathrm{TM}}$ PPLL device, two individual transducers were employed using a clamp. Instead of measuring the tissue displacement across one region, as with the single transducer [2,8], the clamp measured the tissue displacement across the leg. While with two transducers there was some loss of sensitivity, errors due to probe position and choice of echo features on which to lock were eliminated. Also, the reproducibility of measurement was increased compared to one probe, as the placement of one probe caused significant differences in data while with two probes the placements of the two probes varied but still produced the same data.

\section{NIRS model}

Noninvasive NIRS employs the same technology as pulse oximetry to estimate tissue oxygenation. It uses infrared light to penetrate living tissue and estimates tissue oxygenation by measuring the absorption of IR light by tissue chromophores (oxygenated and deoxygenated hemoglobin). The relative absorption of the different wavelengths of the IR light is dependent on the relative concentrations of unoxygenated and oxygenated hemoglobin, which can be determined using a modification of the Beer-Lambert law [10,11]. The NIRS instrument (Somanetics Invos Oximeter, Model 5100c) measured oxygenation in the anterior compartment as described below.

\section{Experimental setup and procedure}

Normal volunteers laid supine on a gurney with one of their legs in the pressure chamber. The leg that was in the pressure chamber was randomized between subjects. The anterior compartment of the leg was then prepared in a sterile fashion. A small region was locally anesthetized with lidocaine and a 14-gauge angiocath was inserted in a caudal to rostral direction at approximately 30 degrees to the skin over the anesthesia site. The needle and catheter were advanced together approximately $4 \mathrm{~cm}$, then the needle was retracted and a slit catheter was inserted in its place for direct IMP measurement. The slit catheter was connected to a syringe of heparinized saline $(2 \mathrm{units} / \mathrm{mL}$, $0.9 \% \mathrm{NaCl}$ injection) through a high pressure line and a pressuretransducing device to prevent the formation of a clot around the tip of the catheter, which could cause unstable IMP readings. The high pressure line was threaded out of the neoprene seal, and the pressuretransducing device was taped to the outside of the pressure chamber level with the slit catheter. The output of the pressure transducer was collected by the transport monitor (Figure 1).

Proper location of the slit catheter was confirmed by gently palpating the anterior compartment and observing a sizeable IMP response on the monitor, as well as asking the subject to dorsiflex his or her foot and observing a similar IMP response. This also ensured that there was no clot at the tip of the catheter.

Standard ultrasound gel was applied to the two transducers of the PPLL clamp, and the clamp was placed on the leg with one transducer in close proximity to the catheter insertion site and the second in the medial part of the calf. The locations of the transducers were adjusted slightly until a clear signal was received from a depth corresponding to the interosseous membrane location [12]. Once the signal stabilized, the pressure chamber was closed.

For each subject, normal baseline measurements were recorded first for 75 seconds. Then, external pressures of 10, 20, 30, 40, 50, 60, and $70 \mathrm{~mm} \mathrm{Hg}$ were applied in a random order. It should be noted at $70 \mathrm{~mm} \mathrm{Hg}$, the compression level was at its upper limit, and the

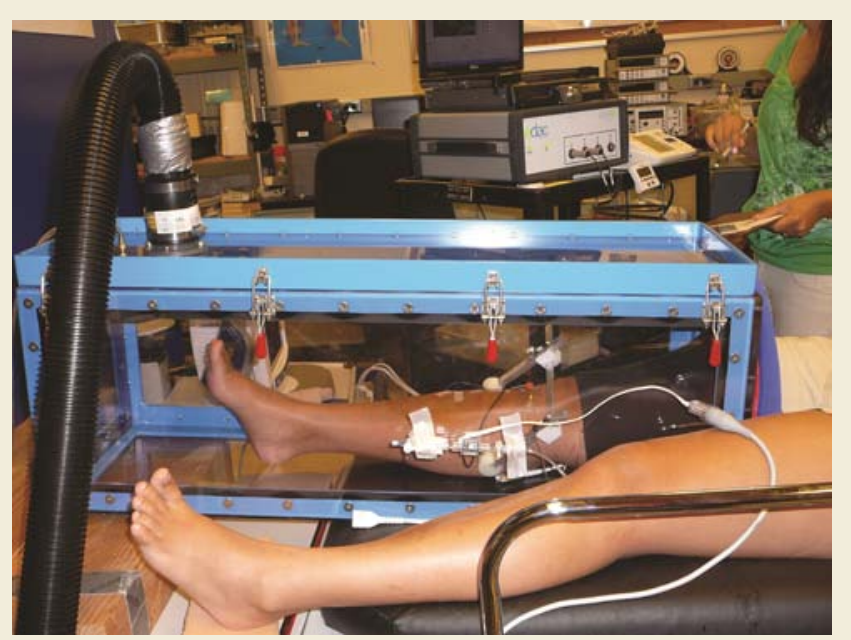

Figure 1: Experimental set up for the PPLL portion of the experiment. The subject lies supine on the gurney with leg up to thigh inserted in the pressure chamber. The slit catheter is inserted into the anterior compartment of the leg and the PPLL probe is attached. 
external pressure in the chamber fluctuated between $67-70 \mathrm{~mm} \mathrm{Hg}$ At each pressure, PPLL measurements were recorded for 75 seconds, and IMP as measured by the slit catheter was noted between 45-50 seconds, after the IMP stabilized. At each pressure level, the subject's blood pressure and heart rate were also taken.

After cycling through each of the pressures, the slit catheter and the PPLL clamp were removed, and NIRS probes were placed on the anterior compartment of both legs. The experiment was then repeated, recording for 2 minutes as baseline, and 60 seconds at each of the pressures.

\section{Data analysis}

Several analyses were performed with the data acquired. First, the relationship between IMP, as measured by slit catheter, and chamber pressure was calculated using a linear fit of plotted data. Second, ANOVAs for both the PPLL and NIRS data for chamber pressure determined whether there was a statistically-significant relationship between IMP and PPLL pulse amplitude, and separately, between IMP and NIRS tissue oxygenation delta. Lastly, a receiver operator characteristic (ROC) curve [13] determined the sensitivity and specificity of the PPLL and NIRS tests, constructed using IMP readings from the slit catheter as a true indicator of ACS.

\section{Results}

In the process of the designing the experiment, the reproducibility of measurement with dPPLL was noted to increase with the clamp compared to one probe, as the placement of one probe caused significant differences in data, while with two probes in a clamp, the placement of the two probes could vary but still produced the same data. In the case of the relationship between the compartment pressure (IMP) and chamber pressure, a linear fit of these data was performed and yielded the following equation: IMP $=11.05+$ $1.11^{\star}$ Chamber Pressure (Figure 2 ). The R [2] value was 0.78 , meaning approximately 78 percent of the variation in the IMP was explained by the chamber pressure applied. The remaining 22 percent was due to unknown variables or inherent variability.

For the PPLL ANOVA, the pulse amplitude was determined at each chamber pressure by measuring a 5 second pulse waveform as monitored by the PPLL, from which heart rate, RMS amplitude, and pulsatility index were extracted. The pulsatility index was a measure of beat-to-beat correlation in the signal, which determined whether the signal was a periodic waveform or random noise. Over the period of recording, the data with a heart rate which did not match the heart rate measured by the blood pressure cuff to within $25 \%$ were rejected as noise. From the remaining waveforms, the data with the highest pulsatility index were used (Figure 3 ). The analysis showed a statistically-significant correlation between the pulse amplitude and chamber pressure $(\mathrm{p}=0.004)$. Subject-to-subject variation was not significant $(\mathrm{p}=0.47)$, suggesting that pulse amplitude did not vary enough between individuals to affect the diagnostic variability of the measurement. For a detailed explanation of the method of analysis, please refer to Lynch and co-workers [3].

As for the NIRS ANOVA, tissue oxygenation delta data were calculated as the average difference in the NIRS tissue oxygenation level for the pressurized leg and the contralateral leg over the last 6 recordings during the test (Figure 4). In the analysis, the tissue oxygenation delta correlated significantly with chamber pressure $(\mathrm{p}=0.003)$. There was also strong subject-to-subject variability in tissue oxygenation delta results $(\mathrm{p}<0.001)$ which may confound the diagnostic accuracy of the NIRS readings.

Slit catheter readings of $30 \mathrm{~mm} \mathrm{Hg}$ or more were considered a true positive indication of compartment syndrome, whereas a reading of less than $30 \mathrm{~mm} \mathrm{Hg}$ was considered a true negative. For the PPLL device, the area under the receiver operator characteristic curve (AUC) was 0.78 , and the sensitivity was 0.75 as was the specificity (Figure 5). As for the NIRS, the AUC was 0.68 , and the sensitivity was 0.65 , as was the specificity (Figure 6 ).

From these analyses, we conclude that the chamber pressure provided a consistent and safe model for inducing a temporary increase in muscle compartment pressure, thus providing a good simulation of an acute compartment syndrome. We can also conclude that both the PPLL pulse amplitudes and NIRS tissue oxygenation readings are significantly correlated to chamber pressure, but that the PPLL pulse amplitude was a slightly better predictor of an acute compartment syndrome as compared to NIRS readings, with less subject-to-subject variability and a higher area under the receiver operator characteristic curve (AUC). The AUC indicates that the PPLL test result from a randomly chosen patient with acute compartment syndrome is more indicative of acute compartment syndrome than that of a patient without acute compartment syndrome, and can be used to compare the differences between the PPLL and NIRS tests [14].

\section{Discussion}

There have been several attempts to develop a reliable instrument for noninvasive estimation of IMP in order to confirm the clinical diagnosis of acute compartment syndrome. One method using NIRS is currently in use, but is unable to be calibrated and may have deficiencies in terms of reliability. Thus, we endeavored to test a noninvasive pulsed phase-locked loop (PPLL) ultrasound device for estimating IMP based on fascial displacement waveforms due to arterial blood pressure pulses. In this regard, we compare PPLL recordings to NIRS, as the latter uses changes in tissue oxygenation to monitor alterations in IMP. We hypothesized that the change in PPLL signal can reliably detect elevated IMP in this model with higher sensitivity and specificity than NIRS. We find that both NIRS and PPLL recordings follow expected results for a simulated acute compartment syndrome with increasing external pressures up to 70 $\mathrm{mm} \mathrm{Hg}$. However, the PPLL technique is a slightly better diagnostic predictor than NIRS with less subject-to-subject variability and a higher area under the receiver operator characteristic curve.

The detection of acute compartment syndrome using the Somanetics NIRS is based on the fact that the body's response to injury is to increase blood flow to the site of the trauma, which NIRS is thought to detect [15]. Thus, the absence of this increase in blood flow suggests hypoperfusion, which could be due to vascular injury or compartment syndrome [4]. The differential light absorption properties detected by the NIRS are then used to solve for the proportion of oxygenated and deoxygenated hemoglobin via the Beer-Lambert law [16].

The digital PPLL is a low-power ultrasound instrument that employs a pulsed phase-locked loop algorithm to detect and continuously monitor very small changes in distance between the ultrasound transducers and subdermal tissue that is capable of reflecting the ultrasonic pulse. Each arterial pulsation causes the compartment to expand transiently. This generates a characteristic 
Citation: Lee SH, Padilla M, Lynch JE, Hargens AR. Noninvasive Measurements of Pressure for Detecting Compartment Syndromes. J Orthopedics Rheumatol. 2013;1(1): 5.

waveform that is recorded in real time and reflects the muscle fascia's accommodation and rebound due to the arterial pressure pulse [8]. Fascial displacements in compartments with elevated IMP are significantly greater than fascial displacements in normal compartments with baseline perfusion pressure [8].

Our acute compartment syndrome model consisted of 15 normal, healthy subjects and external pressures of 10 to $70 \mathrm{~mm} \mathrm{Hg}$ were applied to raise IMP as measured by the gold standard invasive slit catheter. The first analysis performed estimated the relationship between compartment and chamber pressure using a linear fit, yielding an $\mathrm{R}^{2}$ value of 0.78 , meaning 78 percent of the variation in the dependent variable can be explained by the chamber pressure (Figure 2). This analysis documents that our model reliably simulates an acute compartment syndrome.

From our data, analyses of variance (ANOVA) were performed for both the PPLL and NIRS data for each chamber pressure. NIRS data show a significant variation in tissue oxygenation levels at different chamber pressures $(\mathrm{p}=0.003)$, although there is large subjectto-subject variability $(\mathrm{p}<0.001)$ (Figure 3$)$. Likewise, PPLL data show a significant variation in pulse amplitudes at different chamber pressures $(\mathrm{p}=0.004)$, and the subject-to-subject variation is not significant $(\mathrm{p}=0.47)$, suggesting that the individual variation in pulse amplitude does not affect the diagnostic accuracy of the measurement (Figure 4). Receiver operator characteristic (ROC) curves are used to determine the sensitivity and specificity of diagnosing elevated IMP. Sensitivity and specificity for diagnosing normal IMP by the PPLL ( $<30 \mathrm{~mm} \mathrm{Hg}$ from a slit catheter reading) from elevated IMP $(>30 \mathrm{~mm} \mathrm{Hg})$ are 0.75 and 0.75 respectively, and the area under the curve (AUC) is 0.78 (Figure 5). For the NIRS, the sensitivity and specificity are 0.65 and 0.65 , respectively, and the AUC is 0.68 (Figure 6). Since the ROC curve is a two-dimensional depiction of classifier performance, it is easiest to compare to ROC curves by reducing each to a single scalar value representing expected performance, such as the area under the ROC curve (AUC). Random guessing produces a diagonal line with a slope of 1 and an AUC of 0.5, thus the closer to 1 the AUC is, the better the classifier [14].

One of the limitations for the NIRS is the difficulty in finding a consistently reliable position for the NIRS probe on subjects' legs that could give a good baseline measurement. The placement of the NIRS

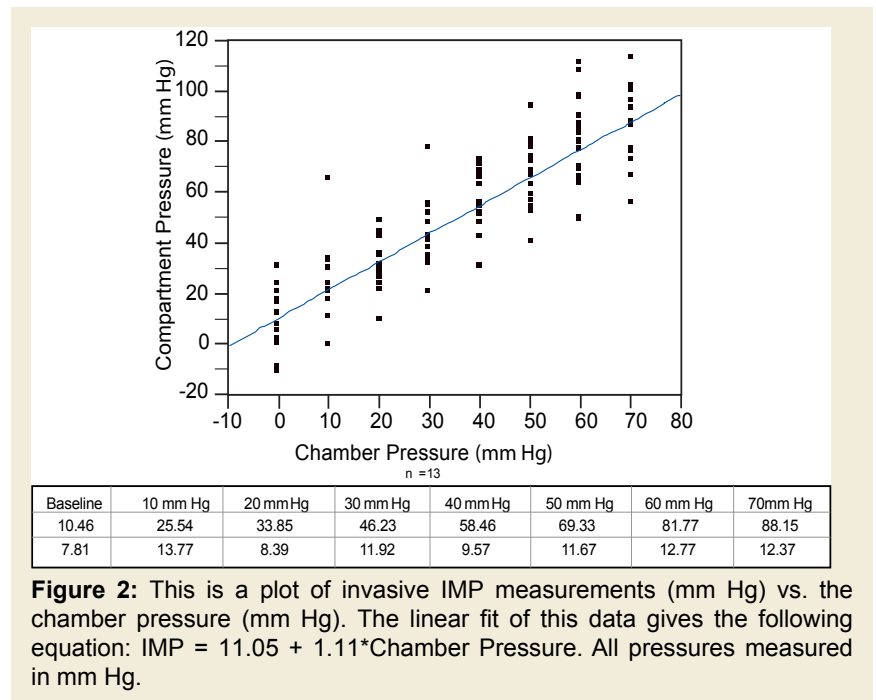

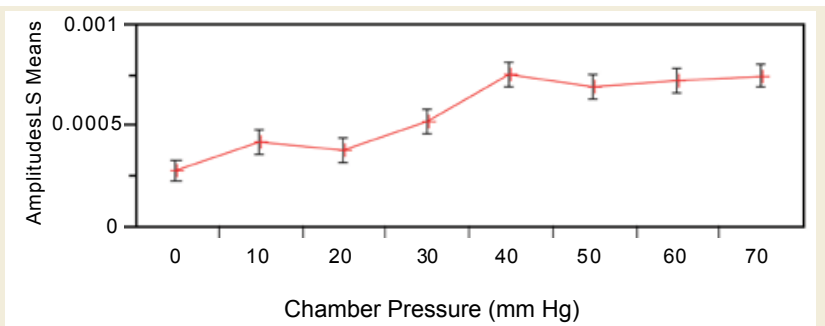

Figure 3: Pulse amplitude measured with the PPLL at different chamber pressures $(\mathrm{mm} \mathrm{Hg})$. In the ANOVA, a statistically significant relationship between pulse amplitude and chamber pressure was found $(p=0.004)$ Subject-to-subject variation in pulse amplitude was not significant $(p=0.47)$ which suggests that pulse amplitude variation between subjects does not vary enough to affect the diagnostic accuracy of the measurement. All pressures measured in $\mathrm{mm} \mathrm{Hg}$

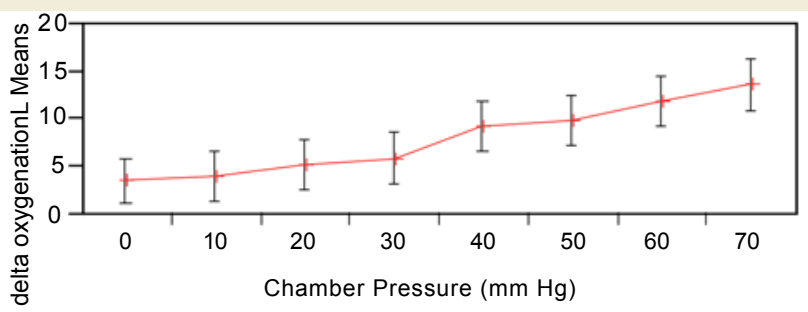

Figure 4: Tissue oxygenation delta measured with NIRS at different chamber pressures $(\mathrm{mm} \mathrm{Hg})$. In the ANOVA, the tissue oxygenation delta (average difference in NIRS tissue oxygenation level for pressurized leg and contralateral leg) was significantly related to chamber pressure $(p=0.003)$. There was also strong subject-to-subject variation in tissue oxygenation deltas $(p<0.001)$ which may affect the diagnostic accuracy of the NIRS reading.

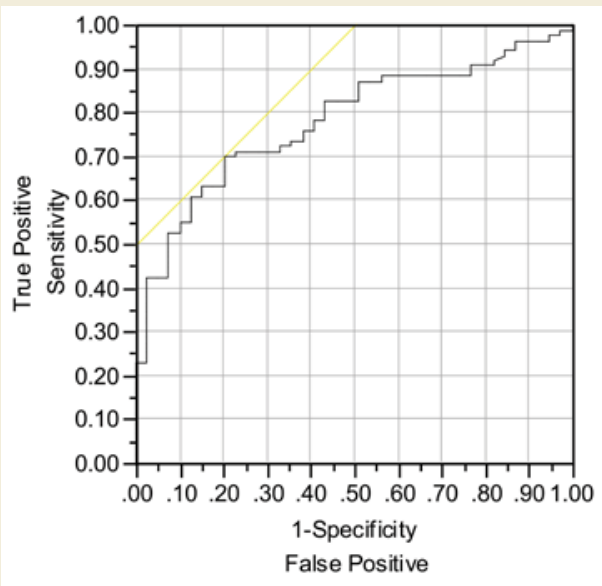

Figure 5: ROC curve for the PPLL. This curve has an AUC of 0.78; at the inflection point sensitivity is approximately 0.75 and the specificity is also approximately 0.75 .

probes in different positions affects which compartment is measured, and their placement at varying distances from the point of trauma can also affect the measurement [17]. However, our short-duration experimental protocol may limit the accuracy of NIRS because the NIRS may take a longer period of time than the PPLL to establish a steady state [15]. In addition, the cycling between different pressures shows rebound hyperemia when moving from a high pressure back down to a lower pressure and this may affect our NIRS results. We plan to address this issue in a future study to establish the amount of 
Citation: Lee SH, Padilla M, Lynch JE, Hargens AR. Noninvasive Measurements of Pressure for Detecting Compartment Syndromes. J Orthopedics Rheumatol. 2013;1(1): 5.

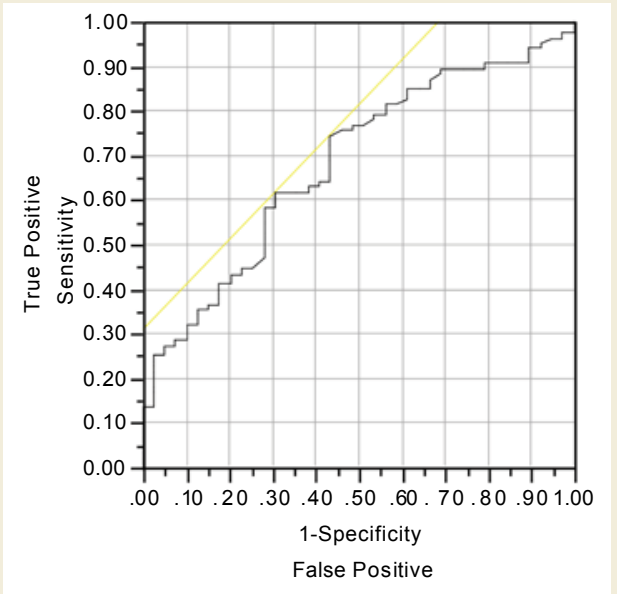

Figure 6: ROC curve for the NIRS. This curve has an AUC of 0.68 ; a the inflection point sensitivity is approximately 0.65 and specificity is also approximately 0.65 .

time it takes for the NIRS to stabilize when a pressure is applied and also the amount of time it takes for NIRS to return to a baseline value. However, it is important to point out that NIRS technology requires a longer period of time to equilibrate to a pressure than the PPLL method [15].

With regards to PPLL, one limitation is due to the placement of the clamp probe. Because the clamp, with two transducers, is much easier to place and obtain a clear signal, two transducers are preferable over a single transducer. However, this makes the signal less specific for the anterior compartment, as it may also detect changes in the superficial and deep posterior compartments. With the transducers arranged in clamp format, it is not possible to isolate single compartments, which is a feature not encountered with the NIRS probes. In this respect, the NIRS may have some advantage over the PPLL.

\section{Conclusion}

Based on the data obtained in this model ACS using normal, healthy volunteers, we conclude that the chamber pressure provides a consistent and safe model for inducing a temporary and accurate increase in intracompartment pressures consistent with an acute compartment syndrome. We also conclude that both the PPLL pulse amplitudes and NIRS tissue oxygenation readings are significantly related to chamber pressure. However, the PPLL device offers a slightly better predictor of acute compartment syndrome than NIRS technology, with somewhat less subject-to-subject variability and greater sensitivity and specificity than the NIRS device. Future directions for this study would include larger study populations as well as expansion to trauma patients in a hospital setting.

\section{References}

1. Hargens AR, Mubarak SJ (1998) Current concepts in the pathophysiology, evaluation, and diagnosis of compartment syndrome. Hand Clin 14: 371-383.

2. Wiemann JM, Ueno T, Leek BT, Yost WT, Schwartz AK, et al. (2006) Noninvasive measurements of intramuscular pressure using pulsed phaselocked loop ultrasound for detecting compartment syndromes: a preliminary report. J Orthop Trauma 20: 458-463.

3. Lynch JE, Heyman JS, Hargens AR (2004) Ultrasonic device for the noninvasive diagnosis of compartment syndrome. Physiol Meas 25: N1-9.
4. Shuler MS, Reisman WM, Kinsey TL, Whitesides TE Jr, Hammerberg EM, et al. (2010) Correlation between muscle oxygenation and compartment pressures in acute compartment syndrome of the leg. J Bone Joint Surg Am 92: 863-870.

5. Lynch JE, Lynch JK, Cole SL, Carter JA, Hargens AR (2009) Noninvasive monitoring of elevated intramuscular pressure in a model compartment syndrome via quantitative fascial motion. J Orthop Res 27: 489-494.

6. Styf J, Wiger P (1998) Abnormally increased intramuscular pressure in human legs: comparison of two experimental models. J Trauma 45: 133-139.

7. Reneman RS, Slaaf DW, Lindbom L, Tangelder GJ, Arfors KE (1980) Muscle blood flow disturbances produced by simultaneously elevated venous and total muscle tissue pressure. Microvasc Res 20: 307-318.

8. Garabekyan T, Murphey GC, Macias BR, Lynch JE, Hargens AR (2009) New noninvasive ultrasound technique for monitoring perfusion pressure in a porcine model of acute compartment syndrome. J Orthop Trauma 23: 186193.

9. Ueno T, Ballard RE, Shuer LM, Cantrell JH, Yost WT, et al. (1998) Noninvasive measurement of pulsatile intracranial pressure using ultrasound. Acta Neurochir Suppl 71: 66-69.

10. Tobias JD, Johnson GA, Rehman S, Fisher R, Caron N (2008) Cerebral oxygenation monitoring using near infrared spectroscopy during one-lung ventilation in adults. J Minim Access Surg 4: 104-107.

11. Yoshitani K, Kawaguchi M, Miura N, Okuno T, Kanoda T, et al. (2007) Effects of hemoglobin concentration, skull thickness, and the area of the cerebrospinal fluid layer on near-infrared spectroscopy measurements. Anesthesiology 106: 458-462.

12. Brahim F, Zaccardelli W (1986) Ultrasound measurement of the anterior leg compartment. Am J Sports Med 14: 300-302.

13. Schalkwyk Jv (2001) The magnificent ROC.

14. Fawcett $T$ (2006) An introduction to ROC analysis. Pattern Recognition Letters 27: 861-874.

15. Shuler MS, Reisman WM, Whitesides TE Jr, Kinsey TL, Hammerberg EM, et al. (2009) Near-infrared spectroscopy in lower extremity trauma. J Bone Joint Surg Am 91: 1360-1368.

16. Fadel PJ, Keller DM, Watanabe H, Raven PB, Thomas GD (2004) Noninvasive assessment of sympathetic vasoconstriction in human and rodent skeletal muscle using near-infrared spectroscopy and Doppler ultrasound. J Appl Physiol 96: 1323-1330.

17. Heckman MM, Whitesides TE Jr, Grewe SR, Rooks MD (1994) Compartment pressure in association with closed tibial fractures. The relationship between tissue pressure, compartment, and the distance from the site of the fracture. J Bone Joint Surg Am 76: 1285-1292.

\section{Acknowledgements}

This work was supported by the National Institute of Arthritis and Musculoskeletal and Skin Diseases through NIH SBIR grant number 2R44AR053750-02A1. 